Article

\title{
The Effects of a Multi-Ingredient Performance Supplement Combined with Resistance Training on Exercise Volume, Muscular Strength, and Body Composition
}

\author{
Dean Directo ${ }^{1, \dagger}$, Michael W.H. Wong ${ }^{1,+}{ }^{+}$, Marcus L. Elam ${ }^{2}$, Paul Falcone ${ }^{1}$, Adam Osmond ${ }^{1}$ and \\ Edward Jo ${ }^{1, *}$ \\ 1 Human Performance Research Laboratory, Department of Kinesiology and Health Promotion, California \\ State University Pomona, Pomona, CA 92805, USA; dean.directo@gmail.com (D.D.); \\ mike.wong203@gmail.com (M.W.H.W.); paul.h.falcone@gmail.com (P.F.); adosmond7@gmail.com (A.O.) \\ 2 Department of Nutrition and Food Science, California State University Pomona, Pomona, CA 92805, USA; \\ mlelam@gmail.com \\ * Correspondence: ejo@cpp.edu; Tel.: +01-909-869-5499 \\ + Co-first authors.
}

Received: 6 June 2019; Accepted: 22 June 2019; Published: 25 June 2019

\begin{abstract}
The effects of a multi-ingredient performance supplement (MIPS) incorporating a mixture of branched chain amino acids, beta-alanine, glutamine, creatine, and piperine on resistance training (RT)-induced adaptations remains unclear. Therefore, the purpose of this study was to investigate the effects of this investigational MIPS during six weeks of RT on performance and body composition. Thirty recreationally trained males and females were recruited for this pair-matched, double-blind, placebo-controlled investigation. Subjects were assigned to consume either an experimental MIPS (MIPS) $(n=15)$ or a placebo (PLA) $(n=15)$ concurrently with a six-week periodized RT program. Body composition, one-repetition maximum (1RM), and muscular power were assessed at pre- and post-training. Weekly relative volume load was compared between groups. The MIPS and PLA groups demonstrated a significant increase in total body mass (MIPS $=+2.9 \pm 1.3 \%$; PLA $=+2.5 \pm 1.7 \%$ ) and lean mass (MIPS $=+5.0 \pm 2.1 \%$; PLA $=+3.1 \pm 1.9 \%)(p<0.001)$ with no changes in fat mass. There were no group $\times$ time interactions for any of the body composition measures. Both groups demonstrated similar improvements in maximum strength for the back squat, bench press, and deadlift as well as lower body power from pre- to post-training $(p<0.001)$. Within the limitations of the current investigation, results failed to demonstrate the benefits of the experimental MIPS for muscular strength and body composition across six weeks of RT compared to PLA.
\end{abstract}

Keywords: creatine; beta-alanine; ergogenic; BCAA; skeletal muscle

\section{Introduction}

A relatively large body of research has been dedicated to investigating methods of potentiating exercise performance and training-induced adaptations. A widely exploited strategy is the use of dietary supplements containing bioactive compounds or nutrients designed specifically to promote an ergogenic response or support metabolic demands during exercise [1]. Concurrent supplementation of dietary performance supplements during resistance training (RT) has gained widespread popularity among competitive and recreational athletes alike due to their purported function of improving exercise quality and augmenting the rate of training adaptations [2-4]. An increasingly popular method of supplementation involves consumption of a single mixture of various substances, commonly referred 
to as a multi-ingredient performance supplement (MIPS). Although MIPS vary widely in ingredient composition, a recent product includes a blend of creatine, beta-alanine, branched-chain amino acids (BCAA), glutamine, and plant-based compounds such as piperine [5-8].

A large proportion of currently available MIPS compounds are implicated as "pre-workout" supplements with the intent of inducing a subsequent acute ergogenic effect via stimulatory ingredients such as caffeine. However, an alternate class of MIPS are designed with the aim of providing metabolic support through nutritional substrate provision for bioenergetic or anabolic processes (i.e., myofibrillar protein synthesis) as well as promotion of skeletal muscle recovery. Regardless of the ongoing controversy of the ideal type of supplements or nutrients for performance enhancement, BCAA, beta-alanine, glutamine, creatine, and piperine have demonstrated a variable degree of ergogenic effects on the human body when consumed individually [9-13]. This has stimulated the adoption of MIPS that incorporate a blend of these substances in efforts to combine the ergogenic properties of each ingredient into one efficient supplement. The multi-ingredient approach to ergogenic supplementation has previously shown to facilitate the physiological adaptations associated with RT [14-16]. However, there is currently limited evidence regarding the efficacy of a MIPS comprised specifically of a mixture of BCAA, creatine, beta-alanine, glutamine, and black pepper fruit extract (piperine) on morphological and functional RT-induced muscular adaptations. Therefore, the purpose of this study was to investigate the effects of a MIPS comprised of the aforementioned ingredients on exercise volume, muscular function, and body composition across six weeks of resistance training.

\section{Materials and Methods}

\subsection{Experimental Approach to the Problem}

A double-blind, placebo-controlled experiment with a mixed between- (treatment group) and within-groups (time) factor design was implemented for this study (Figure 1). Subjects were pair-matched by relative strength and sex and placed into one of two treatment groups for a total of eight weeks with six weeks of RT: experimental MIPS group (MIPS, $n=15$ ) or placebo control group (PLA, $n=15$ ). Baseline descriptive measures are presented in Table 1 . The first week of the experiment incorporated familiarization of performance testing protocols on Monday, baseline assessment of body composition and strength on Wednesday, and baseline assessment of muscular power followed by familiarization of resistance exercises on Friday. For next six weeks, both groups performed RT as detailed below. Concurrently with RT, the MIPS group consumed a multi-ingredient dietary supplement containing a mixture of BCAA, beta-alanine, creatine hydrochloride, piperine, and glutamine while PLA consumed an isocaloric and taste-matched placebo (details below). During the week after the completion of the RT protocol, subjects underwent post-treatment testing procedures. This study was approved by the Institutional Review Board (IRB\#15-0156).

\subsection{Subjects}

Thirty $(n=30)$ healthy college-aged, resistance-trained male $(n=14)$ and female $(n=16)$ subjects were recruited for this study. Prior to participation, each subject signed a document of informed consent. Subjects met the following inclusion criteria: (1) age $=18$ to 32 years, (2) not a competitive athlete in a collegiate or professional sport, and (3) recreationally resistance-trained as defined by resistance exercise performed 3-4 days/week for six months prior to the start of the study and could perform the back squat and bench press with a load 1.0 (male) or 0.75 (female) and 0.75 (male) or 0.5 (female) times their bodyweight, respectively. Subjects were excluded from participation if they reported a medical or surgical history that would have contraindicated the experimental protocol and/or confounded the interpretation of results. In addition, daily use of any dietary supplements within 12 weeks prior to the study called for exclusion. 


\subsection{Resistance Training Protocol}

The RT protocol comprised of three training sessions per week on nonconsecutive days. RT was performed for six weeks following an initial week in which familiarization of the exercises was implemented. During the six-week RT period, Session 1 of each week incorporated the following exercises in the listed order: back squat, bench press, deadlifts, shoulder press, rows, and dumbbell chest press. The back squat, bench press, and deadlift were performed for four sets for 12 repetitions, while the remaining exercises were performed for three sets at 10 repetitions with 10-repetition maximum (RM) loads. Session 2 of each week incorporated the same exercises as Session 1 but excluded the deadlifts. The back squat and bench press were performed for four sets for 10 repetitions while the remaining exercises were performed for three sets for eight repetitions with $8 \mathrm{RM}$ loads. Session 3 of each week consisted of the same exercises as Session 1 in the same order. The back squat, bench press, and deadlift were prescribed at five sets for eight repetitions while remaining exercises were prescribed at three sets for six repetitions with 6RM loads. The initial 10RM loads were determined during the familiarization session in the week before the six-week RT by attempting 10 repetitions with an estimated 10RM and adjusting the load in subsequent attempts based on rating of perceived exertion as presented previously [17]. Initial $8 \mathrm{RM}$ and 6RM loads were estimated based on the 10RM load $(8 \mathrm{RM}=10 \mathrm{lbs}$ more than $10 \mathrm{RM}$ and $6 \mathrm{RM}=20 \mathrm{lbs}$ more than $10 \mathrm{RM})$. For the initial training week, a 1RM-based load prescription was implemented for the back squat, deadlift, and bench press (Session $1=60 \% 1 \mathrm{RM}$, Session $2=65 \% 1 \mathrm{RM}$, and Session $3=70 \% 1 \mathrm{RM})$. Thereafter, training load adjustments were made on a set-to-set and week-to-week basis as described previously $[17,18]$. The final week of training incorporated a taper in which volume was reduced. A certified strength and conditioning specialist monitored all training sessions.

\subsection{Dietary Supplementation Protocols}

The experimental dietary supplement was a proprietary multi-ingredient compound comprised of a blend of BCAA ( $6 \mathrm{~g} / \mathrm{serving}$, 4:1:1 leucine, isoleucine, and valine ratio), L-glutamine ( $3 \mathrm{~g} / \mathrm{serving})$, beta-alanine ( $2 \mathrm{~g} /$ serving), creatine $\mathrm{HCl}(2 \mathrm{~g} /$ serving), and piperine ( $5 \mathrm{mg} /$ serving) (MusclePharm Co., Denver, CO, USA). A single serving provided $1 \mathrm{~g}$ of carbohydrate constituting approximately $5 \mathrm{kcal}$. To test the experimental MIPS under manufacturer dosing recommendations, subjects consumed one serving of their assigned supplement immediately post-workout and before sleep on training days (Monday, Wednesday, and Friday); two separate single servings were taken intersession upon waking and before sleep on Tuesday, Thursday, Saturday, and Sunday. The placebo was a calorie- and taste-matched supplement without the active ingredients listed above, and the same dosing instructions as MIPS were given. The MIPS and placebo were administered in a double-blind manner. Containers for each supplement were labeled " $\mathrm{A}$ " or " $\mathrm{B}$ ". Information regarding the content of supplement " $\mathrm{A}$ " and " $\mathrm{B}$ " was documented by the manufacturer and sealed in an envelope until completion of data analysis.

\subsection{Muscular Strength Testing}

Baseline and post-treatment 1RM strength tests were administered for the back squat, bench press, and deadlift exercises in the listed order. Prior to testing, subjects completed a standardized dynamic warm-up lasting approximately $10 \mathrm{~min}$. Afterwards, the $1 \mathrm{RM}$ test was administered according to procedures previously described by Zourdos et al. [17]. A RT-specific rating of perceived exertion based upon repetitions-in-reserve was used to aid in load selection with successive attempts [17]. A successful attempt was defined according to the standards presented by the International Powerlifting Federation Technical Rulebook [19]. A certified strength and conditioning specialist administered all 1RM tests. 


\subsection{Muscular Power Testing}

Muscular power during the back squat was determined utilizing a linear position transducer (Tendo Power Analyzer, Tendo Sports Machines, Trencin, Slovakia) [20]. The free end of the cable was tethered to the end of the barbell while the transducer was secured to the floor. Subjects performed three 4-repetition trials against a load commensurate to $70 \%$ and $80 \%$ of the subject's baseline 1RM. The trial with the greatest average power across the four repetitions was used for analysis. Subjects were instructed to perform each trial with maximum effort during the concentric phase. Depth of the descent was controlled using an adjustable seat, and eccentric tempo was set at $2 \mathrm{~s}$. Peak power (PWR $\left.\mathrm{PEAK}_{\text {( }}\right)$ and average power (PWR $\mathrm{AVG}_{\mathrm{A}}$ ) were deduced from force, barbell displacement, and time of barbell displacement data using data acquisition software interfaced to the device [Intraclass Correlation Coefficient $(3,1)=0.89,95 \% \mathrm{CI}=0.82-0.93$ ]. Assessment of power was administered $48 \mathrm{~h}$ following the pre- and post-training maximum strength tests.

\subsection{Body Composition Testing}

Body composition was measured by dual-energy X-ray absorptiometry (DXA) (Hologic Discovery-QDR Series Densitometer, Bedford, MA, USA). Fat mass (FM) and non-bone lean mass (LM) (i.e., lean mass - bone mineral content) was analyzed for the whole body. All subjects prior to each scan were free from metallic clothing and accessories. Subjects were tested following first morning void for pre- and post-DXA scans in a fasted state with no food or fluid intake and heavy physical activity prior to the scan. All DXA scans were performed prior to strength tests. The DXA machine was calibrated before each scan using a manufacturer-provided phantom. All DXA measurements and analyses was conducted by a single certified technologist.

\subsection{Dietary Control}

Participants were instructed to avoid any other dietary supplements or ergogenic aids and maintain consistent dietary intake for the duration of the study. Daily dietary intake was monitored using MyFitnessPal (Under Armour Inc., Baltimore, MD, USA). Dietary intake was assessed weekly to ensure that nutrient and caloric intake remained consistent throughout the experimental period.

\subsection{Statistical Analysis}

A 2 (group) $\times 2$ (time) $\times 2$ (sex) analysis of variance (ANOVA) was used for tests of main effects and interactions for all performance and body composition data. In the event of a significant F-ratio, a Bonferroni follow-up test was used for pairwise comparisons. Total relative volume load as calculated by multiplying $1 \mathrm{RM} \%$ load by repetitions were analyzed using an independent $t$-test. All statistical analyses were performed using Statistical Package for Social Science (SPSS, version 14.0 Chicago, IL, USA) with significance set at $p<0.05$. The effects of treatment were calculated as the percent change in performance measures from baseline to post-treatment between MIPS and PLA. Magnitude-based inference analysis, as described previously [21,22], was used to identify clinically meaningful differences in the delta score of each performance measure between treatment groups. The precision of the magnitude inference was set at $90 \%$ confidence limits, using a $p$-value derived from an independent $t$-test comparing mean differences in percent delta change of measures between groups. Threshold values were standard deviations of control group values multiplied by 0.2. Inferences of true differences between PLA and MIPS were determined as beneficial (positive), trivial, or harmful (negative) [22]. Inferences were based on the confidence limit relative to the smallest clinically meaningful effect to be positive, trivial, or negative. Unclear results were reported if the observed confidence interval overlapped both positive and negative values. The probability of the effect was evaluated according to the following scale: $<0.5 \%$, most unlikely; $0.5-5 \%$, very unlikely; 5-25\%, unlikely; 25-75\%, possibly; 75-95\%, likely; $95-99.5 \%$, very likely; $>99.5 \%$, most likely [21]. 


\section{Results}

\subsection{Descriptive Measures}

All baseline descriptive measures are indicated in Table 1. There were no significant differences between MIPS and PLA for any baseline descriptive measures.

Table 1. Between-group comparison of descriptive baseline measures.

\begin{tabular}{|c|c|c|}
\hline Descriptive Measures & $\begin{array}{c}\text { MIPS } \\
(n=15) \\
(7 \mathrm{~m}, 8 \mathrm{f})\end{array}$ & $\begin{array}{c}\text { PLA } \\
(n=15) \\
(7 \mathrm{~m}, 8 \mathrm{f})\end{array}$ \\
\hline Age (years) & $21.9 \pm 1.8$ & $23.3 \pm 1.8$ \\
\hline Total Body Mass (kg) & $63.3 \pm 10.8$ & $66.9 \pm 12.0$ \\
\hline Lean Mass $(\mathrm{kg})$ & $44.9 \pm 9.0$ & $49.0 \pm 10.8$ \\
\hline Fat Mass (kg) & $15.9 \pm 3.9$ & $15.3 \pm 5.4$ \\
\hline Body Fat Percentage (\%) & $25.3 \pm 5.2$ & $22.8 \pm 7.6$ \\
\hline Height $(\mathrm{cm})$ & $165.8 \pm 8.2$ & $168.3 \pm 9.2$ \\
\hline Upper Body Strength $\left(\mathrm{kg} \cdot \mathrm{kg} \mathrm{TBM}^{-1}\right)$ & $1.0 \pm 0.4$ & $1.0 \pm 0.4$ \\
\hline Lower Body Strength $\left(\mathrm{kg} \cdot \mathrm{kg} \mathrm{TBM}^{-1}\right)$ & $1.4 \pm 0.6$ & $1.3 \pm 0.4$ \\
\hline
\end{tabular}

Data presented as mean $\pm \mathrm{SD}$.

\subsection{Body Composition}

Pre- and post-training body composition data and respective $p$-values for main time effects are displayed in Table 2. Both MIPS and PLA groups exhibited a significant increase in total body mass $(\mathrm{TBM})$ and LM $(p<0.001)$ with no group $\times$ time interaction for these variables. There were no main time effects or group $\times$ time interaction for FM. No interaction of sex was detected.

Table 2. Body composition changes across 12 weeks of resistance training in the multi-ingredient performance supplement (MIPS) and placebo (PLA) groups.

\begin{tabular}{ccccc}
\hline Variable & Group & PRE & POST & $\begin{array}{c}\text { Time Effect } \\
(p \text {-Value })\end{array}$ \\
\hline \multirow{2}{*}{ Total Body Mass (kg) } & MIPS & $63.3 \pm 10.8$ & $65.1 \pm 11.2$ & $<0.001$ \\
& PLA & $66.9 \pm 12.0$ & $68.6 \pm 12.7$ & $<0.001$ \\
\hline \multirow{2}{*}{ Lean Mass (kg) } & MIPS & $44.9 \pm 9.0$ & $47.2 \pm 9.6$ & $<0.001$ \\
& PLA & $49.0 \pm 10.8$ & $50.6 \pm 11.4$ & $<0.001$ \\
\hline \multirow{2}{*}{ Fat Mass (kg) } & MIPS & $15.9 \pm 3.9$ & $15.5 \pm 4.0$ & $=0.07$ \\
& PLA & $15.3 \pm 5.4$ & $15.5 \pm 6.0$ & $=0.50$ \\
\hline
\end{tabular}

Data presented as mean \pm SD.

\subsection{Absolute and Relative Maximum Strength}

There was a main time effect indicating a significant 1RM improvement for each exercise for MIPS (back squat $=+26.8 \pm 23.3 \%, p<0.001$; bench press $=+20.5 \pm 13.5 \%, p<0.001$; deadlift $=+27.6 \pm 20.4 \%$, $p<0.001$ ) and PLA (back squat $=+31.4 \pm 24.7 \%, p<0.001$; bench press $=+14.3 \pm 12.0 \%, p<0.001$; deadlift $=+20.4 \pm 14.9 \%, p<0.001$ ) (Figure 1). There was no group $\times$ time interaction for back squat, bench press, or deadlift 1RM. Similar statistical outcomes were found for all 1RM measures normalized to LM (Figure 2). No interaction of sex was found. 


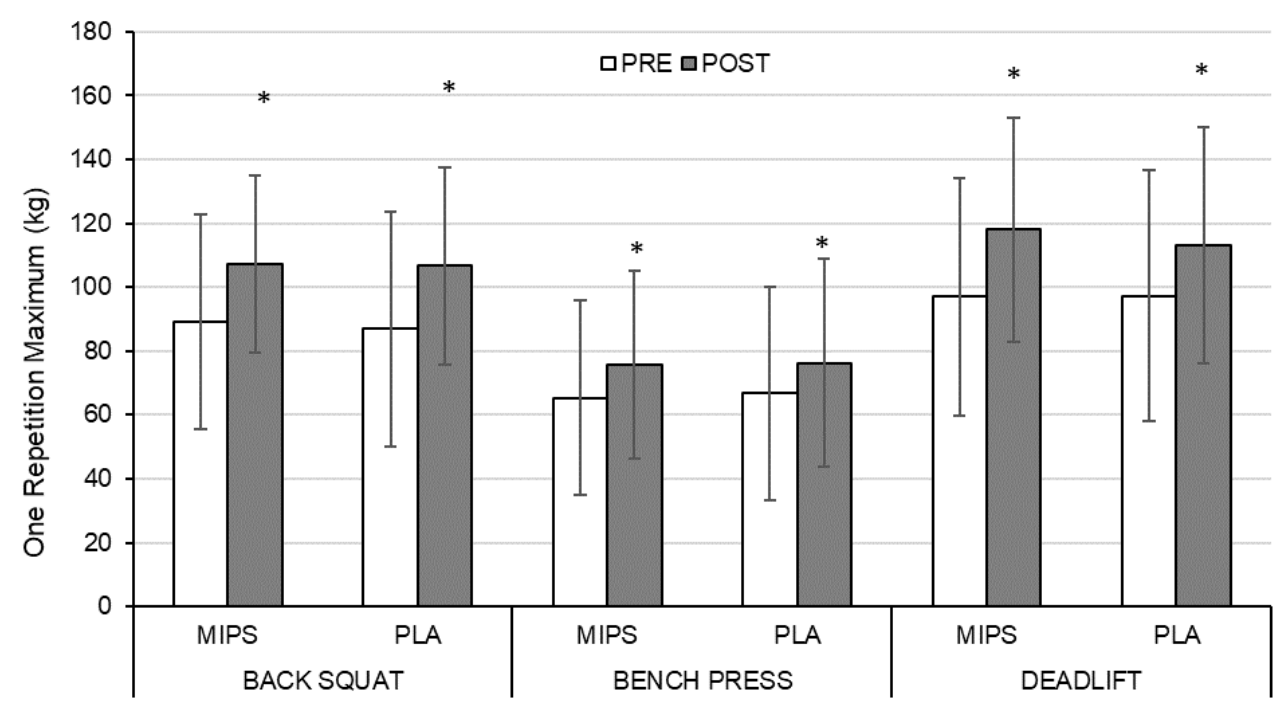

Figure 1. Pre- to post-training relative change in absolute one repetition maximum for back squat, bench press, and deadlift for MIPS and PLA. MIPS = experimental MIPS group, PLA = placebo group. Data presented as mean \pm SD. * Significant pre- to post-training change $(p<0.001)$.

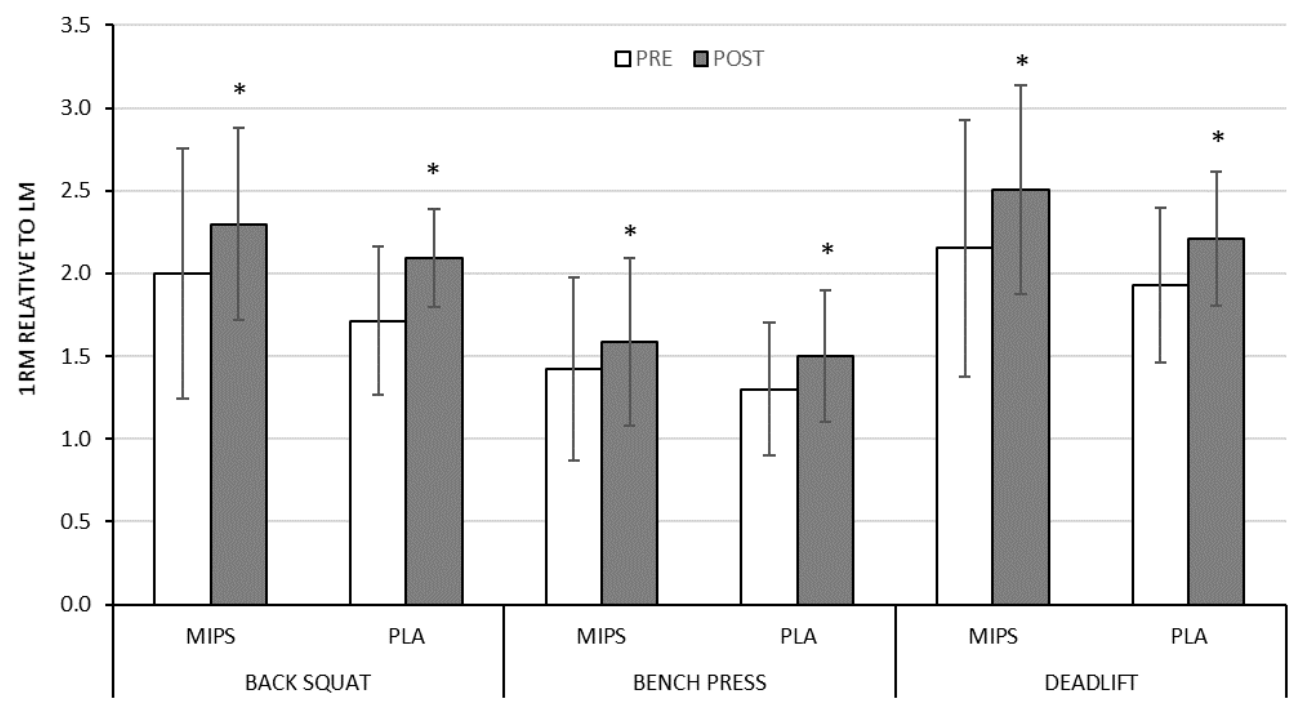

Figure 2. Pre- to post-training relative change in one repetition maximum for back squat, bench press, and deadlift normalized to lean mass for MIPS and PLA. MIPS = experimental MIPS group, PLA $=$ placebo group. Data presented as mean \pm SD. * Significant pre- to post-training change $(p<0.001)$.

Magnitude-based inference analysis indicated that the MIPS treatment is "possibly beneficial" in comparison to PLA treatment with regards to percent delta change in absolute and normalized (to LM) bench press and deadlift strength (Table 3). There were unclear results and therefore uncertain qualitative inferences for the difference in percent delta back squat (absolute and normalized to LM). 
Table 3. Percent change in strength variables from pre- to post-training in MIPS vs. PLA treatments, and qualitative inferences (QI) for the effects of MIPS on each variable.

\begin{tabular}{cccccc}
\hline Variable & $\begin{array}{c}\text { MIPS } \\
\text { (Mean } \pm \text { SD) }\end{array}$ & $\begin{array}{c}\text { PLA } \\
(\text { Mean } \pm \text { SD) }\end{array}$ & $\begin{array}{c}\text { Mean } \\
\text { Difference } \\
(\text { MIPS }- \text { PLA })\end{array}$ & $p$-Value & $\begin{array}{c}\text { QI for Effect Magnitude } \\
\text { (Mean Difference } \pm \text { 90\% CL) }\end{array}$ \\
\hline$\Delta$ Squat 1RM (\%) & $26.8 \pm 24.1$ & $31.4 \pm 25.6$ & -4.6 & 0.62 & $\begin{array}{c}\text { Unclear results } \\
(-4.6 \pm 15.6)\end{array}$ \\
\hline$\Delta$ Bench 1RM (\%) & $20.5 \pm 13.9$ & $14.3 \pm 12.4$ & 6.1 & 0.21 & $\begin{array}{c}\text { Possibly beneficial } \\
(6.1 \pm 8.1)\end{array}$ \\
\hline$\Delta$ DL 1RM (\%) & $27.6 \pm 21.2$ & $20.4 \pm 15.4$ & 7.3 & 0.29 & $\begin{array}{c}\text { Possibly beneficial } \\
(7.3 \pm 11.5)\end{array}$ \\
\hline$\Delta$ Squat/LM (\%) & $20.9 \pm 23.6$ & $27.4 \pm 23.7$ & -6.5 & 0.46 & $\begin{array}{c}\text { Unclear results } \\
(-6.5 \pm 13.8)\end{array}$ \\
\hline$\Delta$ Bench/LM (\%) & $14.8 \pm 13.0$ & $11.0 \pm 12.7$ & 3.7 & 0.43 & $\begin{array}{c}\text { Possibly beneficial } \\
(3.7 \pm 7.9)\end{array}$ \\
\hline$\Delta$ DL/LM (\%) & $21.6 \pm 20.1$ & $16.7 \pm 14.4$ & 4.8 & 0.46 & $\begin{array}{c}\text { Possibly beneficial } \\
(4.8 \pm 10.9)\end{array}$ \\
\hline
\end{tabular}

$\mathrm{CL}=$ Confidence Limit, $\mathrm{LM}=$ Lean Mass.

\subsection{Lower Body Power}

There was no group $\times$ time interaction for lower and upper body peak or average power with loads corresponding to $70 \%$ or $80 \% 1 \mathrm{RM}$ (Table 4 ). There was a main time effect in which post-hoc tests showed significant improvements in each measure of lower body power in both MIPS and PLA. There was no interaction of sex.

Table 4. Changes in lower body muscular power across 12 week of resistance training in MIPS and PLA.

\begin{tabular}{ccccc}
\hline \multicolumn{1}{c}{ Variable } & Group & PRE & POST & $\begin{array}{c}\text { Time Effect } \\
(\boldsymbol{p} \text {-Value })\end{array}$ \\
\hline \multirow{2}{*}{$70 \% \mathrm{PWR}_{\mathrm{AVG}}(\mathrm{W})$} & MIPS & $416.6 \pm 148.5$ & $478.6 \pm 151.1$ & $<0.001$ \\
& PLA & $431.2 \pm 166.4$ & $500.7 \pm 162.0$ & $<0.001$ \\
\hline \multirow{2}{*}{$70 \% \mathrm{PWR}_{\text {PEAK }}(\mathrm{W})$} & MIPS & $901.2 \pm 356.7$ & $1129.2 \pm 477.9$ & $=0.001$ \\
& PLA & $1031.1 \pm 433.8$ & $1211.2 \pm 392.0$ & $<0.001$ \\
\hline \multirow{2}{*}{$80 \% \mathrm{PWR}_{\mathrm{AVG}}(\mathrm{W})$} & MIPS & $416.6 \pm 136.0$ & $500.4 \pm 161.1$ & $<0.001$ \\
& PLA & $439.1 \pm 147.1$ & $490.5 \pm 153.9$ & $=0.006$ \\
\hline \multirow{2}{*}{$80 \% \mathrm{PWR}_{\text {PEAK }}(\mathrm{W})$} & MIPS & $963.1 \pm 419.1$ & $1216.3 \pm 508.3$ & $=0.002$ \\
& PLA & $1060.3 \pm 378.6$ & $1241.9 \pm 355.9$ & $=0.002$ \\
\hline
\end{tabular}

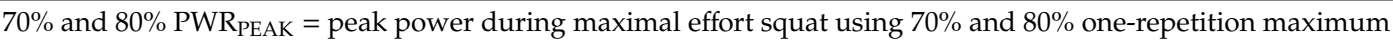
(RM) load, respectively. $70 \%$ and $80 \% \mathrm{PWR}_{\mathrm{AVG}}=$ average power during maximal effort squat using $70 \%$ and $80 \%$ $1 \mathrm{RM}$ load, respectively. PRE $=$ pre-training measurement, $\mathrm{POST}=$ post-training measurement. Data presented as mean \pm SD.

\subsection{Relative Volume Load}

There were no group differences for relative volume load each week and average weekly relative volume load (Figure 3). There was no interaction of sex. 


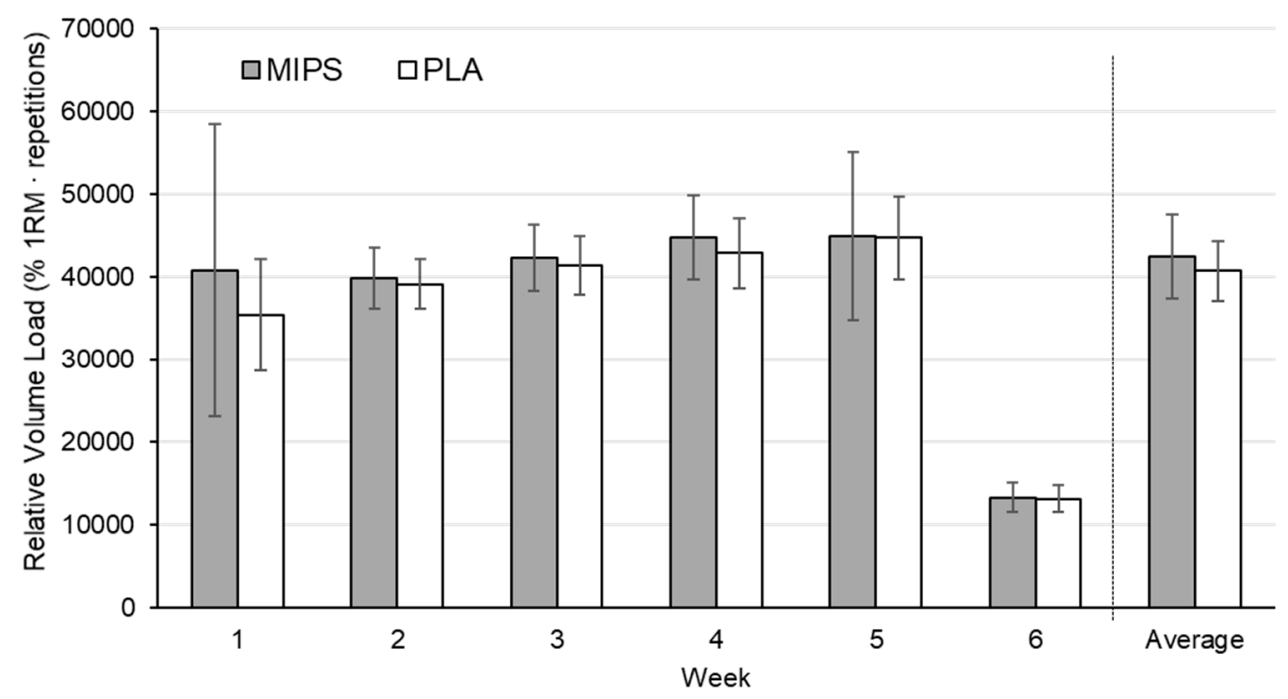

Figure 3. Weekly and average relative volume load between MIPS and PLA. MIPS = experimental MIPS group. PLA = placebo group. Data presented as mean \pm SD.

\subsection{Dietary Intake}

Two-way ANOVA (2 groups $\times 6$ weeks) revealed no significant main effect or group $\times$ time interactions for average daily caloric, protein, carbohydrate $(\mathrm{CHO})$, or fat intake. Total average daily intake did not differ between groups for caloric (MIPS $=1628.4 \pm 159.3 \mathrm{kcals} /$ day vs. PLA $=1708.0 \pm 133.8 \mathrm{kcals} /$ day), $\mathrm{CHO}(\mathrm{MIPS}=175.7 \pm 19.3 \mathrm{~g} /$ day vs. $\mathrm{PLA}=181.1 \pm 16.2 \mathrm{~g} /$ day $)$, protein $(\mathrm{MIPS}=81.7 \pm 11.3 \mathrm{~g} / \mathrm{day}$ vs $\mathrm{PLA}=85.9 \pm 9.5 \mathrm{~g} /$ day), or fat (MIPS $=57.3 \pm 6.4 \mathrm{~g} /$ day vs. PLA $=60.2 \pm 5.4 \mathrm{~g} /$ day) intakes. Within each group, average daily caloric and macronutrient intake per week did not differ among the experimental weeks. There were no interactions of sex.

\section{Discussion}

In summary of the principal findings, daily supplementation of the experimental MIPS failed to elicit any effects on body composition or performance changes across six weeks of RT in comparison to a placebo control. No observable augmentation to weekly and overall training volume was observed with the experimental MIPS treatment, thereby, demonstrating the absence of acute resistance exercise performance benefits. Arguably, the fundamental purpose of MIPS use is to acutely enhance performance such that improved quality of each training bout may ultimately facilitate muscular adaptations. The experimental MIPS, when used as a "post-workout" or "recovery" supplement, is proposed to facilitate these ergogenic effects through increased provision of energy substrates to aid muscle bioenergetics and amino acids to support muscle recovery and protein anabolism. Since the experimental MIPS failed to enhance the quality of each training session as reflected by similar training volume achievements as the placebo group, it would be expected that any RT-induced adaptations would be comparable between treatment groups. The analogous performance gains between treatment groups may be corroborated by the lack of difference in training volume.

The current results regarding training volume are consistent with that of Ormsbee et al. [5] in which authors reported similar outcomes following experimentation with a comparable MIPS mixture. As with the present study, they indicated significant effects of time for upper and lower body strength, however with no group $\times$ time interactions, indicating that the experimental MIPS failed to acutely enhance the overall work achieved during each RT bout. Furthermore, Chromiak et al. [23] and Beck et al. [24] arrived at similar conclusions with physically active (1-5 h of weekly strenuous exercise on a regular basis) and untrained males, respectively. In contrast to the afore-mentioned studies, Kreipke et al. [25] found that their experimental MIPS treatment produced significantly greater volume in squat, deadlift, and bench press exercises compared to the placebo group. In addition, Shelmadine et 
al. [14] and Lowery et al. [26] also reported enhancements in upper body strength with their respective MIPS treatment. However, it is important to note that Lowery et al. [26] employed an eight-week RT program which was two more weeks than that of the present study thereby allowing for an increased window for potential divergences in strength gains to occur. It is also important to emphasize that Shelmadine et al. [14] utilized untrained males while Kreipke et al. [25] and Lowery et al. [26] examined resistance-trained males. Due to the inverse relationship that exists between training status and rate of training progression (i.e., strength increases), there could conceivably be a larger magnitude of effect with untrained subjects [27]. Aside from training status, the composition of MIPS varied across studies with slightly different ingredients and dosages. For example, caffeine, which has previously shown to acutely improve exercise performance, is a notable ingredient absent from the present MIPS but commonly seen in others [1]. Despite the variability across studies regarding MIPS composition, the findings from those studies, in large, point to a key link between training volume and muscular performance enhancement. Specifically, an effective MIPS should fundamentally enhance the quality of each training bout thereby facilitating the rate of training adaptations.

Despite the inclusion of ingredients with some evidential support for ergogenic efficacy, the current MIPS failed to produce a detectable effect on acute performance and RT adaptations. For example, supplementation of beta-alanine in conjunction with RT has shown to attenuate fatigue with consequent increases in training volume compared to a placebo in resistance-trained males [28]. Furthermore, a separate study examining creatine in isolation as well as creatine in combination with beta-alanine found greater strength improvements in both treatment groups compared to a placebo also in resistance-trained males [29]. Despite the inclusion of apparently effective ergogenic ingredients, the nature of our study may not have provided the most favorable conditions for their effects to manifest. For example, a recent position stand on beta-alanine from the International Society of Sports Nutrition suggests that a loading phase of about four weeks is essential for subsequent increase in muscle carnosine levels which is the connecting mechanism between beta-alanine supplementation and performance enhancement [10]. Additionally, a prior review of literature recommends dosages of 2-3 g/day of creatine to increase intramuscular creatine and phosphocreatine stores over a 3-4 weeks period [1]. Thus, it appears that a 3-4-week loading period may be necessary before any effects could occur. Therefore, the length of our experimental timeline may not have provided a sufficient period for ergogenic effects to take place. The results of Lowery et al. [26] seem to support this theory since they observed a positive outcome for upper body strength with their MIPS treatment while utilizing an eight-week RT protocol.

The multi-ingredient approach to performance supplementation certainly presents with limitations in both research and practice. From a research perspective, many studies involving MIPS, such as the present investigation, utilize a pre-engineered blend of ingredients that may or may not in isolation have evidence demonstrating efficacy. In cases even where a MIPS contains evidence-based ingredients, the doses provided in a recommended serving of the supplement may be sub-optimal for a given ingredient and/or individual. This is an inherent limitation in all MIPS research. Moreover, when examining the body of research of single-ingredient performance supplements, it is commonplace to observe relatively large inter-subject variability and a comparable number of positive, negative, and non- responders even in a generally homogenous cohort. This often precludes a statistically detectable effect and keeps the likelihood of even a small worthwhile effect to a minimum. The multi-ingredient approach, even with evidence-supported ingredients, fails to amend these highly variable responses and/or small effects generally associated with dietary supplementation. From a practical application standpoint, athletes or exercising individuals often combine the use of multiple supplements in efforts to acquire a synergistic enhancement of one or more performance attributes. Although synergies between or among single-ingredient supplements are evident, such as between sodium bicarbonate and beta-alanine or caffeine [30-32], there are no known data suggesting a potential synergy among any of the ingredients in the present investigational MIPS. Thus, currently there is no clear justification for the application of the current MIPS at least within the setting and population represented by this 
study. The multi-ingredient strategy in performance supplementation certainly appears logical and at times empirically justified, but the current literature on MIPS does not provide adequate practical information that can properly guide athletes in making evidence-based decisions about how and if MIPS should be consumed.

Strictly within the limits of the present investigation, the overall findings fail to support the benefits of the experimental MIPS in conjunction with a six-week periodized RT program. As an executive summary of findings, supplementation of the experimental MIPS compound did not augment total weekly and overall volume load which likely explains the absence of detectable effects on training adaptations. The body of evidence regarding MIPS use are highly mixed. Given the evidential ergogenic benefits of key individual ingredients namely, creatine and beta-alanine, it is possible that the present investigational MIPS may elicit an effect on performance and/or body composition under different circumstances or populations compared to those applied in the current investigation. Due to the varying combinations of ingredients within the multitude of MIPS compounds, it remains difficult to ascertain superior compounds or optimum combinations and dosages of each ingredient. In the case of examining multi-ingredient supplements, a study design that incorporates individual and combined uses of the products of interest would be integral to enhancing the current understanding of the interactions among individual substances and the practical use of MIPS in sport and exercise. Accordingly, a paradigm shift in MIPS research away from a simple between-group comparison of a MIPS product vs. placebo and towards examining interactions and synergies among single-ingredient supplements would be warranted. As a follow up to the current investigation, it is evident considering the highlighted limitations in MIPS research that a step in reverse, so to speak, is strongly recommended to better justify the combined use of the various ingredients.

Author Contributions: Co-first authors, D.D. and M.W.H.W. designed the study, collected and analyzed data and prepared the manuscript. M.L.E. and P.F. assisted with the study design and manuscript preparation. A.O. assisted with data collection. E.J. served as the principle investigator and oversaw the study.

Funding: This study was funded in part by the International Society of Sport Nutrition Research Grant.

Conflicts of Interest: The authors declare no conflict of interest.

\section{References}

1. Kreider, R.B.; Wilborn, C.D.; Taylor, L.; Almada, A.L.; Collins, R.; Cooke, M.; Earnest, C.P.; Greenwood, M.; Kalman, D.S.; Kerksick, C.M.; et al. ISSN exercise \& sport nutrition review: Research \& recommendations. J. Int. Soc. Sports Nutr. 2010, 7,7.

2. Young, C.R.; Stephens, M.B. Sports and nutritional supplement use in USMC recruits: A pilot study. Mil. Med. 2009, 174, 158-161. [CrossRef] [PubMed]

3. Massad, S.J.; Shier, N.W.; Koceja, D.M.; Ellis, N.T. High school athletes and nutritional supplements: A study of knowledge and use. Int. J. Sport Nutr. 1995, 5, 232-245. [CrossRef] [PubMed]

4. Erdman, K.A.; Fung, T.S.; Doyle-Baker, P.K.; Verhoef, M.J.; Reimer, R.A. Dietary supplementation of high-performance Canadian athletes by age and gender. Clin. J. Sport Med. 2007, 17, 458-464. [CrossRef] [PubMed]

5. Ormsbee, M.J.; Mandler, W.K.; Thomas, D.D.; Ward, E.G.; Kinsey, A.W.; Simonavice, E.; Panton, L.B.; Kim, J.S. The effects of six weeks of supplementation with multi-ingredient performance supplements and resistance training on anabolic hormones, body composition, strength, and power in resistance-trained men. J. Int. Soc. Sports Nutr. 2012, 9, 49. [CrossRef] [PubMed]

6. Willems, M.E.; Sallis, C.W.; Haskell, J.A. Effects of multi-ingredient supplementation on resistance training in young males. J. Hum. Kinet. 2012, 33, 91-101. [CrossRef] [PubMed]

7. Spillane, M.; Schwarz, N.; Willoughby, D.S. Heavy resistance training and peri-exercise ingestion of a multi-ingredient ergogenic nutritional supplement in males: Effects on body composition, muscle performance and markers of muscle protein synthesis. J. Sports Sci. Med. 2014, 13, 894-903. 
8. Outlaw, J.J.; Wilborn, C.D.; Smith-Ryan, A.E.; Hayward, S.E.; Urbina, S.L.; Taylor, L.W.; Foster, C.A. Acute effects of a commercially-available pre-workout supplement on markers of training: A double-blind study. J. Int. Soc. Sports Nutr. 2014, 11, 40. [CrossRef]

9. Blomstrand, E.; Eliasson, J.; Karlsson, H.K.; Köhnke, R. Branched-chain amino acids activate key enzymes in protein synthesis after physical exercise. J. Nutr. 2006, 136, 269S-273S. [CrossRef]

10. Trexler, E.T.; Smith-Ryan, A.E.; Stout, J.R.; Hoffman, J.R.; Wilborn, C.D.; Sale, C.; Kreider, R.B.; Jäger, R.; Earnest, C.P.; Bannock, L.; et al. International society of sports nutrition position stand: Beta-Alanine. J. Int. Soc. Sports Nutr. 2015, 12, 30. [CrossRef]

11. Antonio, J.; Street, C. Glutamine: A potentially useful supplement for athletes. Can. J. Appl. Physiol. 1999, 24, 1-14. [CrossRef]

12. Kreider, R.B. Effects of creatine supplementation on performance and training adaptations. Mol. Cell Biochem. 2003, 244, 89-94. [CrossRef] [PubMed]

13. Srinivasan, K. Black pepper and its pungent principle-piperine: A review of diverse physiological effects. Crit. Rev. Food Sci. Nutr. 2007, 47, 735-748. [CrossRef]

14. Shelmadine, B.; Cooke, M.; Buford, T.; Hudson, G.; Redd, L.; Leutholtz, B.; Willoughby, D.S. Effects of 28 days of resistance exercise and consuming a commercially available pre-workout supplement, NO-Shotgun(R), on body composition, muscle strength and mass, markers of satellite cell activation, and clinical safety markers in males. J. Int. Soc. Sports Nutr. 2009, 6, 16. [CrossRef]

15. Smith, A.E.; Fukuda, D.H.; Kendall, K.L.; Stout, J.R. The effects of a pre-workout supplement containing caffeine, creatine, and amino acids during three weeks of high-intensity exercise on aerobic and anaerobic performance. J. Int. Soc. Sports Nutr. 2010, 7, 10. [CrossRef] [PubMed]

16. Spillane, M.; Schwarz, N.; Leddy, S.; Correa, T.; Minter, M.; Longoria, V.; Willoughby, D.S. Effects of 28 days of resistance exercise while consuming commercially available pre- and post-workout supplements, NO-Shotgun ${ }^{\circledR}$ and NO-Synthesize ${ }^{\circledR}$ on body composition, muscle strength and mass, markers of protein synthesis, and clinical safety markers in males. Nutr. Metab. (Lond.) 2011, 8, 78. [PubMed]

17. Zourdos, M.C.; Klemp, A.; Dolan, C.; Quiles, J.M.; Schau, K.A.; Jo, E.; Helms, E.; Esgro, B.; Duncan, S.; Garcia Merino, S.; et al. Novel Resistance Training-Specific Rating of Perceived Exertion Scale Measuring Repetitions in Reserve. J. Strength Cond Res. 2016, 30, 267-275. [CrossRef] [PubMed]

18. Klemp, A.; Dolan, C.; Quiles, J.M.; Blanco, R.; Zoeller, R.F.; Graves, B.S.; Zourdos, M.C. Volume-equated highand low-repetition daily undulating programming strategies produce similar hypertrophy and strength adaptations. Appl. Physiol. Nutr. Metab. 2016, 41, 699-705. [CrossRef]

19. IPF. International Powerlifting Federation Technical Rules Book 2016; IPF: Luxembourg, 2016.

20. Shimano, T.; Kraemer, W.J.; Spiering, B.A.; Volek, J.S.; Hatfield, D.L.; Silvestre, R.; Vingren, J.L.; Fragala, M.S.; Maresh, C.M.; Fleck, S.J.; et al. Relationship between the number of repetitions and selected percentages of one repetition maximum in free weight exercises in trained and untrained men. J. Strength Cond. Res. 2006, 20, 819-823.

21. Hopkins, W.G.; Marshall, S.W.; Batterham, A.M.; Hanin, J. Progressive statistics for studies in sports medicine and exercise science. Med. Sci. Sports Exerc. 2009, 41, 3-13. [CrossRef]

22. Batterham, A.M.; Hopkins, W.G. Making meaningful inferences about magnitudes. Int. J. Sports Physiol. Perform. 2006, 1, 50-57. [CrossRef] [PubMed]

23. Chromiak, J.A.; Smedley, B.; Carpenter, W.; Brown, R.; Koh, Y.S.; Lamberth, J.G.; Joe, L.A.; Abadie, B.R.; Altorfer, G. Effect of a 10-week strength training program and recovery drink on body composition, muscular strength and endurance, and anaerobic power and capacity. Nutrition 2004, 20, 420-427. [CrossRef] [PubMed]

24. Beck, T.W.; Housh, T.J.; Johnson, G.O.; Coburn, J.W.; Malek, M.H.; Cramer, J.T. Effects of a drink containing creatine, amino acids, and protein combined with ten weeks of resistance training on body composition, strength, and anaerobic performance. J. Strength Cond. Res. 2007, 21, 100-104. [PubMed]

25. Kreipke, V.C.; Allman, B.R.; Kinsey, A.W.; Moffatt, R.J.; Hickner, R.C.; Ormsbee, M.J. Impact of Four Weeks of a Multi-Ingredient Performance Supplement on Muscular Strength, Body Composition, and Anabolic Hormones in Resistance-Trained Young Men. J. Strength Cond. Res. 2015, 29, 3453-3465. [CrossRef] [PubMed]

26. Lowery, R.P.; Joy, J.M.; Dudeck, J.E.; de Souza, E.O.; McCleary, S.A.; Wells, S.; Wildman, R.; Wilson, J.M. Effects of 8 weeks of $X$ pand $^{\circledR} 2 X$ pre workout supplementation on skeletal muscle hypertrophy, lean body mass, and strength in resistance trained males. J. Int. Soc. Sports Nutr. 2013, 10, 44. [CrossRef] [PubMed] 
27. Fleck, S.; Kraemer, W. Resistance Training and Exercise Prescription. Designing Resistance Training Programs; Human Kinetics: Champaign, IL, USA, 2004; pp. 81-179.

28. Hoffman, J.R.; Ratamess, N.A.; Faigenbaum, A.D.; Ross, R.; Kang, J.; Stout, J.R.; Wise, J.A. Short-duration beta-alanine supplementation increases training volume and reduces subjective feelings of fatigue in college football players. Nutr. Res. 2008, 28, 31-35. [CrossRef] [PubMed]

29. Hoffman, J.; Ratamess, N.; Kang, J.; Mangine, G.; Faigenbaum, A.; Stout, J. Effect of creatine and beta-alanine supplementation on performance and endocrine responses in strength/power athletes. Int. J. Sport Nutr. Exerc. Metab. 2006, 16, 430-446. [CrossRef] [PubMed]

30. Tobias, G.; Benatti, F.B.; de Salles Painelli, V.; Roschel, H.; Gualano, B.; Sale, C.; Harris, R.C.; Lancha, A.H., Jr.; Artioli, G.G. Additive effects of beta-alanine and sodium bicarbonate on upper-body intermittent performance. Amino. Acids. 2013, 45, 309-317. [CrossRef] [PubMed]

31. Bellinger, P.M.; Howe, S.T.; Shing, C.M.; Fell, J.W. Effect of combined $\beta$-alanine and sodium bicarbonate supplementation on cycling performance. Med. Sci. Sports Exerc. 2012, 44, 1545-1551. [CrossRef]

32. Felippe, L.C.; Lopes-Silva, J.P.; Bertuzzi, R.; McGinley, C.; Lima-Silva, A.E. Separate and Combined Effects of Caffeine and Sodium-Bicarbonate Intake on Judo Performance. Int. J. Sports Physiol. Perform. 2016, 11, 221-226. [CrossRef]

(C) 2019 by the authors. Licensee MDPI, Basel, Switzerland. This article is an open access article distributed under the terms and conditions of the Creative Commons Attribution (CC BY) license (http://creativecommons.org/licenses/by/4.0/). 